\title{
Myasthenia Class IV
}

National Cancer Institute

\section{Source}

National Cancer Institute. Myasthenia Class IV. NCI Thesaurus. Code C112017.

Severe weakness affecting non-ocular muscles. May also have ocular muscle weakness. 